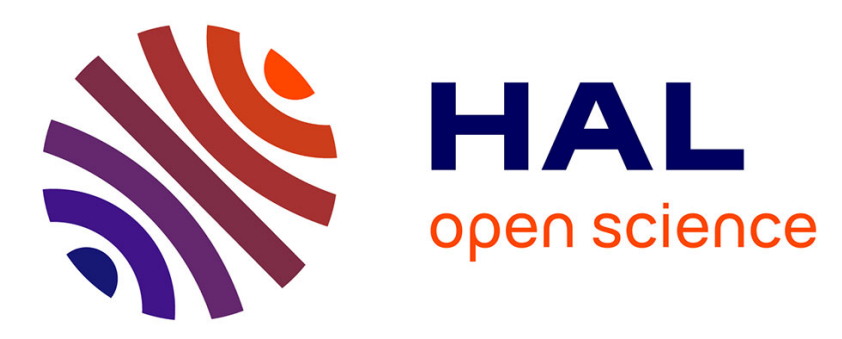

\title{
Enhanced-transmission metamaterials as anisotropic plates
}

\author{
F.I. Baida, M. Boutria, R. Oussaid, D. van Labeke
}

\section{To cite this version:}

F.I. Baida, M. Boutria, R. Oussaid, D. van Labeke. Enhanced-transmission metamaterials as anisotropic plates. Physical Review B: Condensed Matter and Materials Physics (1998-2015), 2011, 84 (3), pp.035107. 10.1103/PhysRevB.84.035107 . hal-00655052

\section{HAL Id: hal-00655052 https://hal.science/hal-00655052}

Submitted on 21 Apr 2021

HAL is a multi-disciplinary open access archive for the deposit and dissemination of scientific research documents, whether they are published or not. The documents may come from teaching and research institutions in France or abroad, or from public or private research centers.
L'archive ouverte pluridisciplinaire HAL, est destinée au dépôt et à la diffusion de documents scientifiques de niveau recherche, publiés ou non, émanant des établissements d'enseignement et de recherche français ou étrangers, des laboratoires publics ou privés. 


\title{
Enhanced-transmission metamaterials as anisotropic plates
}

\author{
F. I. Baida, ${ }^{1, *}$ M. Boutria,,${ }^{1,2}$ R. Oussaid, ${ }^{2}$ and D. Van Labeke ${ }^{1}$ \\ ${ }^{1}$ Département d'Optique P.M. Duffieux, Institut FEMTO-ST UMR 6174 CNRS Université de Franche-Comté, \\ F-25030 Besançon Cedex, France \\ ${ }^{2}$ Université des Sciences et de la Technologie Houari Boumedienne, Algeria
}

(Received 8 March 2011; revised manuscript received 6 May 2011; published 18 July 2011)

\begin{abstract}
We present an original design of anisotropic metamaterial plates exhibiting extraordinary transmission through perfectly conductor metallic screens perforated by a subwavelength double-pattern rectangular aperture array. The polarization properties of the fundamental guided mode inside the apertures are at the origin of the anisotropy. The metal thickness is a key parameter that is adjusted in order to get the desired value of the phase difference between the two transversal electromagnetic field components. As an example, we treat the case of a half-wave plate having $92 \%$ transmission coefficient. Such a study can be easily extended to design anisotropic plates operating in terahertz or microwave domains.
\end{abstract}

DOI: 10.1103/PhysRevB.84.035107

PACS number(s): 42.15.Eq, 42.60.Da, 42.82.Et

\section{INTRODUCTION}

Naturally birefringent materials, such as quartz, are usually needed to design quarter- and half-wave plates. In the optical domain such materials exhibit small index values (around 1.5), leading to an efficient transmission coefficient through the plates. In addition, the ordinary and extraordinary indices difference is very small and this leads to relatively thick plates (a hundred or a thousand of wavelengths for a half-wave plate). Contrarily, in the terahertz domain, the birefringence becomes larger $\left(5 \times 10^{-2}\right.$ at $1 \mathrm{THz}$ for quartz $\left.{ }^{1}\right)$ but the average optical index of the material is also increased $(2.13$ at $1 \mathrm{THz}$ for the same crystal). This obviously leads to a high reflection coefficient and thus to very weak transmission signals. The transmission is also decreased by intrinsic absorption losses of the used materials ${ }^{1}$ in the corresponding spectral range.

Recently, a quarter-wave plate for terahertz applications has been proposed by Saha et al.. It is based on artificial anisotropy between TE and TM polarizations created by diffraction through a dielectric lamellar grating. ${ }^{2}$ Polarization properties of hole arrays have been studied in many works. In 2004, Gordon et al. have observed strong polarization dependence in the transmission through elliptical nanohole arrays. ${ }^{3}$ More recently, a similar phenomenon was demonstrated through asymmetric $\mathrm{C}$-shaped holes. ${ }^{4}$ Optical rotation was experimentally demonstrated by using a metallic film perforated by an array of chiral hole structures: Archimedean spiral slots that induce polarization rotation in the far infrared region ${ }^{5}$ while Bai et al. ${ }^{6}$ used gammadion-shaped cavities in the visible and near infrared.

In Ref. 7, a polarization conversion was observed for near-infrared wavelengths, in transmission mode through a trilayer structure (metal/dielectric/metal) with L-shaped hole arrays. More recently, an optical polarization manipulation has been theoretically studied by a "stereo-plasmonic" structure made of three metallic layers perforated with a periodic array of rectangular holes. ${ }^{8}$ To obtain the polarization properties, the rectangular apertures are differently orientated in each layer.

The aim of our study is to present original design of a half-wave plate (or a quarter-wave one) for the terahertz domain. A geometrical anisotropy is caused by a grating of rectangular subwavelength apertures engraved into a thin perfectly conducting film. As it is well known, a monoperiodic (1D) metallic lamellar grating with a period smaller than $\lambda$ behaves as a perfect polarizer (the transmitted plane wave is perpendicularly polarized to the grating grooves). Similar polarization properties are obtained for a metallic film perforated by a biperiodic (2D) square array of rectangular apertures: an electric field perpendicular to the longer side of the apertures is totally transmitted while a field parallel to this side is completely reflected. ${ }^{9}$ This is directly linked to the excitation and the propagation, at a given frequency, of a guided mode along the metal thickness. Nevertheless, one notes that the transmission greatly depends on this thickness. ${ }^{10}$ In fact, interference harmonics of this guided mode lead to additional peaks in the transmission spectrum. This property is general for any structure where the transmission is based on the excitation and the propagation of a guided mode. It was already verified in the case of annular apertures ${ }^{11}$ and for bowtie nanoapertures. ${ }^{12}$

As it will be seen in the following, these polarization and propagation properties of the fundamental guided mode can be exploited to conceive a structure where anisotropy is induced by the presence of two different polarization sensitive patterns: two perpendicular rectangular apertures of unequal lengths as seen in Fig. 1. The idea is that each of the two transverse components of the electric field is effectively transmitted by only one aperture at the same wavelength. A phase difference appears because the effective index of the guided mode differs from one to the other aperture. Consequently, it is possible to adjust the value of this phase difference by changing the metal layer thickness.

In the following, we give a simple equation which explains the principle of the proposed structure, the way to obtain a half-wave plate and how to design it through the determination of its geometrical parameters. Then, more realistic numerical simulations based on the finite difference in the time domain (FDTD) algorithm are performed in order to obtain more exact values of the parameters and to verify that the desired polarization properties can be correctly obtained. 


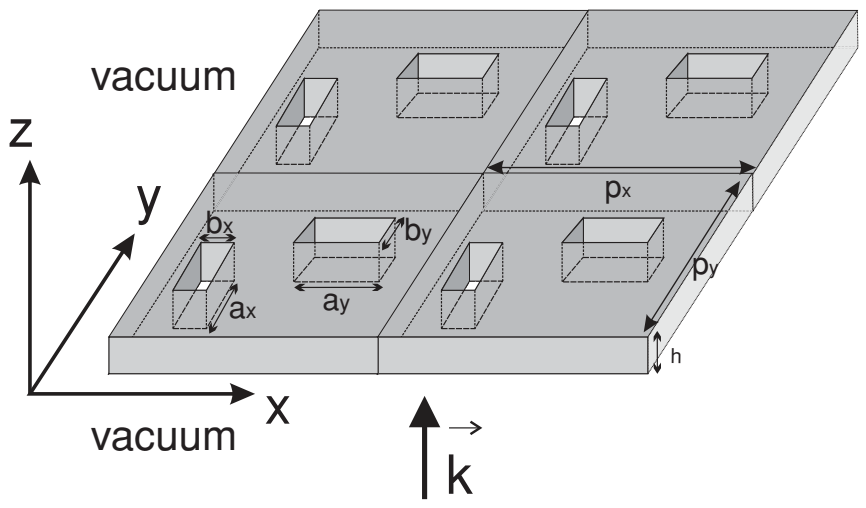

FIG. 1. Schematic of the proposed structure: a biperiodic grating ( $p_{x}$ and $p_{y}$ are the periods along $x$ and $y$, respectively) with two rectangular apertures per period $\left(a_{x} \neq a_{y}\right)$ engraved into a $h$-thick metallic screen.

\section{MONOMODAL MODAL METHOD (MMM)}

The structure is a thin metallic film (thickness $h$ ) perforated by a square $2 \mathrm{D}$ grating (period $p_{x}=p_{y}=p$ ) of rectangular apertures (see Fig. 1). For simplicity, all the considered structures are supposed to be free standing in vacuum and there is no dielectric media in the cavities (apertures). The grating period is supposed to be smaller than all the incident wavelengths $(p<\lambda)$ and the angle of incidence is always equal to zero (normal incidence). Consequently, there is only one homogeneous transmitted wave, the zero-order diffracted one which propagates perpendicular to the plate. All the other transmitted diffracted orders are evanescent and are not far-field detected.

In the following, the metal is supposed to be perfectly conducting so no absorption losses are considered. This assumption is justified in the terahertz frequency domain and it is reinforced by the fact that involved propagation distances are small compared to the wavelength $(h<\lambda)$. ${ }^{13}$ The transmission properties of such apertured metallic structures are directly connected to the electromagnetic modes which can propagate in the cavities. In our case, the rectangular apertures are smaller than the incident wavelength and only the fundamental cavity mode can be efficiently excited.

For a rectangular aperture of length $a$ and width $b(b<$ $a / 2$ ), the fundamental mode is the $\mathrm{TE}_{10}$ mode which is polarized perpendicular to the longer side of the aperture. This mode has a cut-off wavelength of $\lambda_{c}=2 a$, which leads to a dispersive effective index verifying the equation:

$$
\widetilde{n}(a)=\sqrt{1-\frac{\lambda^{2}}{\lambda_{c}^{2}(a)}} .
$$

In the proposed structure, there are two perpendicular rectangular cavities. The polarization directions of the modes are perpendicular and the effective index depends on the cavity parameters (the lengths $a_{x}$ and $a_{y}$ in our case). Therefore, by adjusting the length of the two apertures, it is possible to get a geometrical birefringence. Nevertheless, the transmission through each aperture should be maximum for the same value of the wavelength (working point). This last condition is then fulfilled by adjusting the metal thickness.
The transmission properties of apertured metallic structures can be theoretically studied by several methods. The finite elements method and FDTD are very efficient tools but they lead only to numerical calculations. On the contrary, many papers have used the modal method to study the diffraction of electromagnetic waves by periodic structures made in a perfect metal. It is impossible to give an exhaustive bibliography on this subject; however, the formalism is clearly exposed for inductive grids in Ref. 15. The same method is used to study the transmission by an annular apertures array made in perfect conductor. ${ }^{16}$ More recently, in the context of enhanced transmission by small apertures, a review paper by GarcíaVidal et al. describes the method, gives a bibliography, and shows many interesting applications. ${ }^{17}$

In the regions above and below the metal, the incident and diffracted fields are expressed as a series of Rayleigh waves. In the cavities, the field is generally written as a superposition of infinite series of the waveguide modes. Each waveguide mode satisfies both the Helmholtz equation and the appropriate boundary conditions along the vertical walls of the cavities. The tangential components of the electric and magnetic fields are then matched at the grating free space boundaries $(z=0$ and $z=h$ ) which leads to a system of linear equations of infinite dimension.

When the transversal spatial dimensions of the apertures are small, only the fundamental mode of the waveguides has a significant contribution to the diffracted amplitudes. In this case, the linear system can be drastically simplified and a very simple analytical expression for the diffracted field amplitudes can be extracted. This approach, named the monomodal modal method approximation, was presented many years ago in several papers, for instance, in Ref. 15. In the context of enhanced transmission, the monomodal approximation was firstly used for the study of lamellar grating. ${ }^{18-20}$

Within the single-mode approximation, the zero-order complex transmitted amplitude can be written:

$$
t_{0}=\frac{4 Y_{\mathrm{inc}} Y_{m}\left|S_{\mathrm{inc}}\right|^{2} \exp (i \Psi)}{\left(Y_{m}+\widetilde{Y}\right)^{2}-\exp (i 2 \Psi)\left(Y_{m}-\widetilde{Y}\right)^{2}} .
$$

In this equation, $i=\sqrt{-1}, \Psi$ is a phase angle created by wave propagation in the cavity. It depends on the effective index $\widetilde{n}$ of the cavity mode and on the metal thickness $h$ :

$$
\Psi=h \frac{2 \pi}{\lambda} \widetilde{n} .
$$

$Y_{m}$ and $Y_{\text {inc }}$ are the admittances of the cavity mode and of the incident wave, respectively. Let us define $S_{d}$ as an overlap integral. It is an integral over one aperture of the product of the transversal electric fields of the mode and of a Rayleigh wave " $d$ ":

$$
S_{d}=\iint_{\text {Aperture }} \boldsymbol{E}_{m}^{T}\left(\boldsymbol{E}_{d}^{T}\right)^{*} d x d y .
$$

$S_{\text {inc }}$ becomes the corresponding integral $S_{d}$ for the incident wave. $\widetilde{Y}$ is an effective admittance in which all the diffraction effects are incorporated: $\widetilde{Y}=\sum_{d} Y_{d}\left|S_{d}\right|^{2}$. 
In the expression of $\widetilde{Y}$, the summation runs over all the diffractive orders $(p, q \in \mathbb{Z})$ and over the polarization index ( $\sigma=1$, TE Rayleigh mode, $\sigma=2$, TM),

$$
\begin{aligned}
\tilde{Y}= & \sum_{\sigma=1,2} \sum_{p=-\infty}^{+\infty} \sum_{q=-\infty}^{+\infty} Y_{p, q}\left|S_{p, q}\right|^{2}=Y_{\mathrm{inc}}\left|S_{\mathrm{inc}}\right|^{2} \\
& +\sum_{\sigma=1,2} \sum_{p \neq 0} \sum_{q \neq 0} Y_{p, q}\left|S_{p, q}\right|^{2}=Y_{\mathrm{inc}}\left|S_{\mathrm{inc}}\right|^{2}+i C(\lambda)
\end{aligned}
$$

The first term corresponds to the incident mode (diffraction order $p=0, q=0$ ) and it is a real term. All the other terms are pure imaginary because, when $\lambda>p$, all the diffracted orders are evanescent waves and their admittances are pure imaginary numbers. Notations aside, Eq. (2) is given in Ref. 21, a paper that proposes a discussion of the effective medium theory of holey films.

When the apertures have a simple geometry (rectangle, square, disk, annular), the cavity modes have simple mathematical forms. Analytical expressions can then be found for the overlap integral. ${ }^{22}$ Moreover, in the metamaterial limit $(\lambda \gg p)$ all the diffraction terms can be neglected $[C(\lambda) \ll$ $\left.Y_{\text {inc }}\left|S_{\text {inc }}\right|^{2}\right]$ and a very simple equation is obtained:

$$
\begin{aligned}
t_{0} \longrightarrow t_{0 h} & \cong \frac{1}{\cos \Psi+\text { if } \sin \Psi} \text { with } \\
f & =\frac{1}{2}\left(\frac{1}{Y_{\mathrm{inc}}} \frac{Y_{m}}{\left|S_{\mathrm{inc}}\right|^{2}}+\frac{\left|S_{\mathrm{inc}}\right|^{2}}{Y_{m}} Y_{\mathrm{inc}}\right) .
\end{aligned}
$$

Equation (6) leads to an Airy-like formula for the transmitted intensity $T_{0}=\left|t_{0}\right|^{2}$, which is obtained in the theory of the Fabry-Perot interferometer. However, contrarily to a conventional Fabry-Perot interferometer, the effective index in Eq. (3) verifies a dispersion equation with a cutoff. The position of the transmission's maxima seems to be easily determined via Eq. (6). If $\sin \Psi=0(\Psi=l \pi$ with $l$ an integer $\geqslant 0)$, $T_{0}=1$ and transmission maxima are reached.

As we are interested by polarization problems, two kinds of maxima have to be distinguished: they correspond to even or odd values of $l$ :

$$
l \text { odd: } l=1,3,5 \sin \left(\Psi_{x}\right)=0, \cos \left(\Psi_{x}\right)=-1 \Rightarrow t_{x}=-1,
$$

$l$ even: $l=0,2,4,6 \sin \left(\Psi_{x}\right)=0, \cos \left(\Psi_{x}\right)=+1 \Rightarrow t_{x}=1$.

For $l>0$, we obtain a series of maxima similar to FabryPerot's. They depend on metal thickness and they need a sufficient thickness $\left(h>l \frac{\lambda}{2}\right)$ to occur. The solution with $l=0$ is not possible for a conventional Fabry-Perot with a nondispersive index. But it corresponds here to a new kind of maximum. The $l=0$ maximum is reached near the cut-off wavelength. It is important to note that the corresponding value of the wavelength is independent of the metal thickness and can be observed even for a very small thickness. This new transmission peak will be named "cut-off peak" in the following. It was predicted by the coworkers in a theoretical study of coaxial aperture structures in the optical domain ${ }^{11}$ and experimentally observed in Refs. 23 and 24.
The position of the peaks can thus be determined approximatively:

$$
\lambda_{0} \cong \lambda_{\text {cutoff }} ; \lambda_{l>0} \cong \frac{2 h l}{\sqrt{1+\left(\frac{2 h l}{\lambda_{\text {cutoff }}}\right)^{2}}}=\frac{2 h l}{\sqrt{1+\left(h \frac{l}{a}\right)^{2}}} .
$$

As explicitly mentioned in Eq. (1), for a fixed value of the wavelength, the effective index can be controlled by changing the geometrical parameters of the cavity section, for instance, the rectangle length $a$.

The principle of the determination of the parameters of a half-wave plate is now simple to understand. We use a structure (see Fig. 1) with two perpendicular rectangular apertures in the primitive grating cell. The length $a_{x}$ of the first aperture $\left(a_{x}, b_{x} ; a_{x}>b_{x}\right)$ is parallel to the $y$ axis while the length $a_{y}$ of the second aperture $\left(a_{y}, b_{y} ; a_{y}>b_{y}\right)$ is parallel to the $x$ axis. Then, we have to adjust $a_{y}$ in order to have the "cut-off peak" for $y$ polarization which coincides with the $l=1$ peak of the $x$ polarization.

This leads to the structural relation:

$$
a_{y}=\frac{h}{\sqrt{1+\frac{h^{2}}{a_{x}^{2}}}} .
$$

Unfortunately, this last expression does not work correctly because it is found on Airy expression of the transmitted coefficient which is an approximation [Eq. (6)]. It assumes to completely neglect diffraction and it corresponds to an homogenization of the structured plates that is absolutely valid for $\lambda \gg p$. But when $\lambda>p$, the evanescent waves, which are confined in the near field, have a definite influence on the far-field transmitted amplitude as they induce an additional imaginary part for $\widetilde{Y}$. Note that the real part is independent of $\lambda\left(\operatorname{Re} \tilde{Y}=Y_{\text {inc }}\left|S_{\text {inc }}\right|^{2}=\frac{2 \sqrt{2}}{\pi} \sqrt{\frac{a b}{p_{x} p_{y}}}\right.$ for a rectangular aperture array illuminated at normal incidence) but the imaginary part is not equal to zero and vanishes for a wavelength close to the cutoff.

Figure 2 illustrates this point where a rectangular aperture with $a=0.9 p, b=0.1 p$, and $h=p$ is considered. On Fig. 2(a), we have compared the exact transmission $\left(T_{0}=\left|t_{0}\right|^{2}\right)$ and the approximate one $T_{0 h}=\left|t_{0 h}\right|^{2}$. For the two curves, the cut-off peak is obtained for $\lambda \simeq \lambda_{c}=2 a$, but in fact a small shift appears. However, it is clear that the difference between the two curves is larger for the first Fabry-Perot peak $(l=1)$. As shown in Fig. 2(b), the imaginary part of $\widetilde{Y}$ is at the origin of this discrepancy as it induces a red-shift of this peak as mentioned above. However, for the rigorous coefficient $t_{0}$, the phase difference between the cut-off peak and the first Fabry-Perot peak remains around $\pi$.

So, a half-wave plate can be designed but the parameters must be determined with the nonapproximate expression $t_{0}$ [Eq. (2)] or a more general tool such as FDTD that is able to simulate the transmission through a double-apertured structure.

\section{STUDY OF THE WHOLE STRUCTURE}

To design the double structure, we have to take into account the fact that both of the two apertures should have 

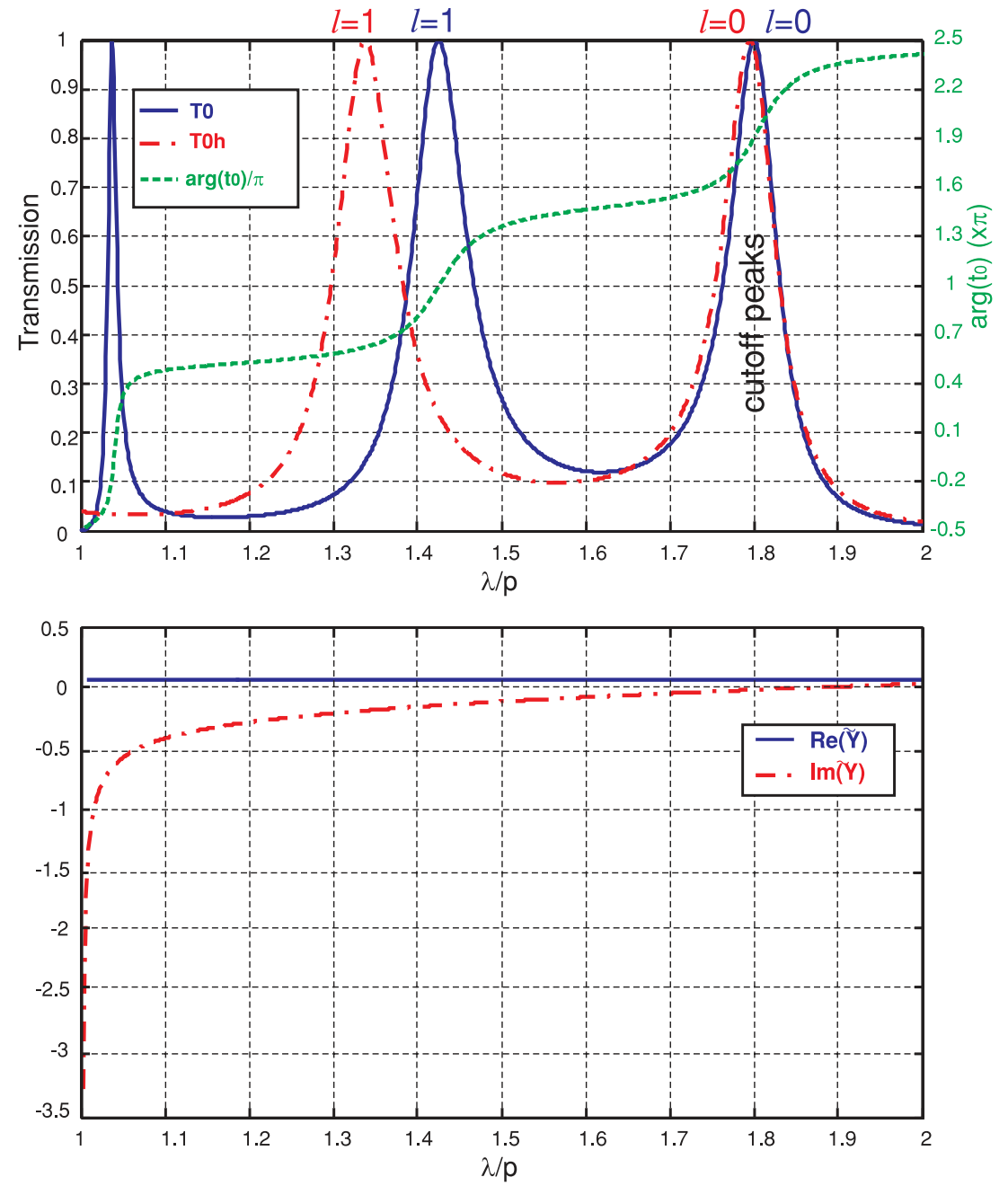

FIG. 2. (Color online) (a) Normalized transmission $T_{0}=\left|t_{0}\right|^{2}$ (solid blue line) and $T_{0 h}=$ $\left|t_{0 h}\right|^{2}$ (in dashed-dotted red line) calculated from Eqs. (2) and (6) for a 2D grating of rectangular apertures with $a=0.9 p, b=0.1 p$, and $h=p$. The phase of $t_{0}$ is also shown (in green dashed line). (b) Real (solid blue line) and imaginary (dashed-dotted red line) parts of the function $\widetilde{Y}$ that contribute in the expression of $t_{0}$. cut-off wavelengths larger than the period. Consequently, $a_{x}$ and $a_{y}$ must be larger that $p / 2$. Thus, we first fix the geometrical parameters of one of the two rectangular apertures, for example, $a_{x}=0.75 p, b_{x}=0.1 p$, and $h=0.8 p$ (see Fig. 1). In addition, $b_{y}<p-a_{x}$ is a second condition that must be fulfilled to enable positioning the two perpendicular rectangular apertures into one grating period. The rest of the optimization procedure is done by varying the length $a_{y}$ and by monitoring both the transmission and the phase difference (PD) using the fast calculation algorithm of the monomodal method.

Let us denote $t_{x}$ and $t_{y}$ the transmission amplitudes through the two apertures $\left(a_{x}, b_{x}\right)$ and $\left(a_{y}, b_{y}\right)$ respectively. Figure 3 shows the result of the optimization steps: we have plotted the PD defined by PD $=\arg \left(t_{y}\right)-\arg \left(t_{x}\right)$ as a function of $\lambda / p$ and $a_{y} / p$. In this figure, three contour plots are underlined: the white lines that correspond to equality of the transmission amplitudes $\left(\left|t_{x}\right|=\left|t_{y}\right|\right)$, the other lines correspond to a specific values of PD: black line corresponds to a PD of $\pi$ and the blue line to $\mathrm{PD}=\pi / 2$.

Consequently, by exploiting the diagram of Fig. 3, one can determine all the geometrical parameters of the desired structure. Two results are summarized in Fig. 3: point A corresponds to a half-wave plate with the geometrical parameters $a_{x}=0.75 p, a_{y}=0.582 p, b_{x}=0.1 p, b_{y}=0.2 p$, and $h=0.8 p$. The operating wavelength is then $\lambda=1.194 p$ with a transmission coefficient of $T_{\lambda / 2}=0.93$ [see Fig. 4(a)]. Point $\mathrm{B}$ corresponds to a quarter-wave plate where the parameters become $a_{x}=0.75 p, a_{y}=0.653 p, b_{x}=0.1 p, b_{y}=0.2 p$, and $h=0.8 p$. The operating wavelength is $\lambda=1.182 p$ in this case but a smaller transmission coefficient $\left(T_{\lambda / 4}=0.57\right)$ is reached as seen in Fig. 4(b).

Let us study more precisely the case of the half-wave plate. The monomodal method can hardly handle the doubleapertured structure. Thus, a finite difference time domain (3DFDTD) home-made code ${ }^{25}$ is used to simulate the transmission through the whole structure. In the $x-y$ plane, the calculation window is equal to the elementary square cell $(p \times p)$ with periodic boundary conditions. In the $z$ direction, perfectly matched layers (PML) boundary conditions are used. A uniform meshing of $\delta_{x}=\delta_{y}=\delta_{z}=p / 150$ is applied, leading to more than $12 \times 10^{6}$ nodes for the whole calculation.

Because of the spatial meshing of the structure, the geometrical parameters of the modeled one must be slightly modified in order to get the same transmission coefficients (peaks position) as the monomodal method (point A of Fig. 3). For example, the metal thickness was found to be $h=0.83 p$ instead of $h=0.8 p$ used within the monomodal method. Accordingly, we have performed three successive steps for the FDTD calculations. Figure 5 presents the results of these 


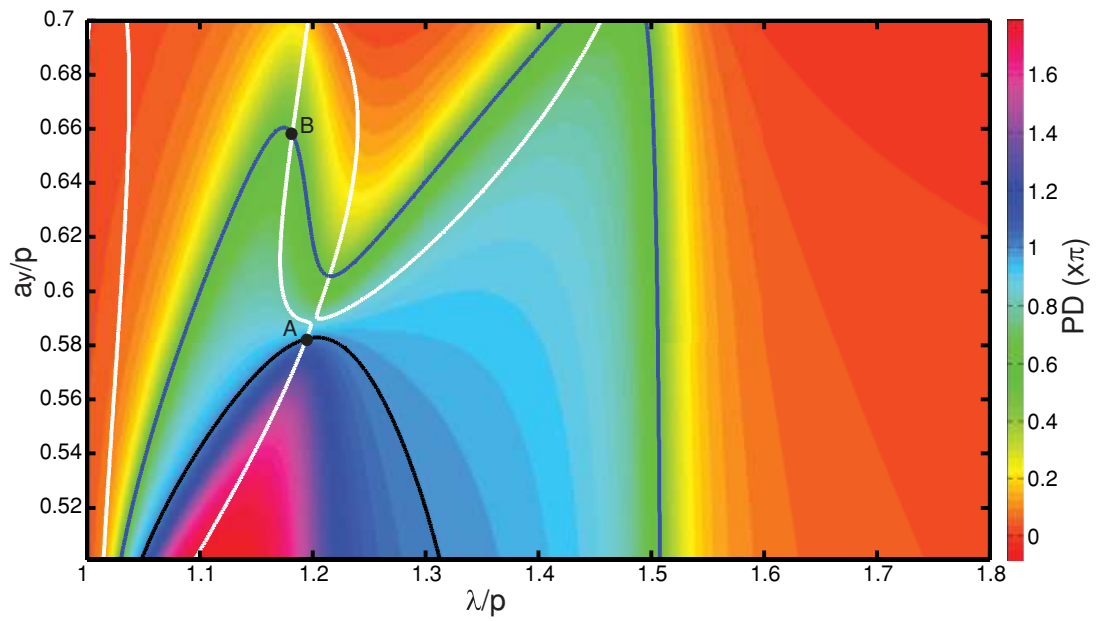

FIG. 3. (Color) Phase difference PD $=$ $\arg \left(t_{y} / t_{x}\right)$ as a function of $\lambda / p$ and $a_{y} / p$ for $h=0.8 p$. The white contour plots give the couples $\left(\lambda, a_{y}\right)$ that correspond to an equal transmission coefficient through the two apertures $\left(\left|t_{x}\right|=\left|t_{y}\right|\right)$. The black line corresponds to $\mathrm{PD}=$ $\pi$ (point $\mathrm{A}$ answers the case of a half-wave plate) and the blue line to $\mathrm{PD}=\pi / 2$ (point $\mathrm{B}$ corresponds to a quarter-wave plate).

calculations in term of transmission spectra obtained through three structures. An $x$-polarized incident plane wave is supposed to illuminate the first grating [Fig. 5(a)] while a $y$-polarized one is considered for the second grating [Fig. 5(b)]. The corresponding transmission spectra are plotted in Figs. 5(d) and 5(e), respectively. One can clearly see that the high transmission at the $\operatorname{FP}(l=1)$ peak of the first spectrum almost corresponds to the cut-off transmission peak of the second structure. A small shift is necessary to adjust the PD between the transmitted $x$ and $y$ components. The lower parts of Fig. 5 verify that the complete structure, with two perpendicular apertures per period, has the properties of a half-wave plate. The transmission intensity spectrum is calculated in the case of a normally incident plane wave but linearly polarized

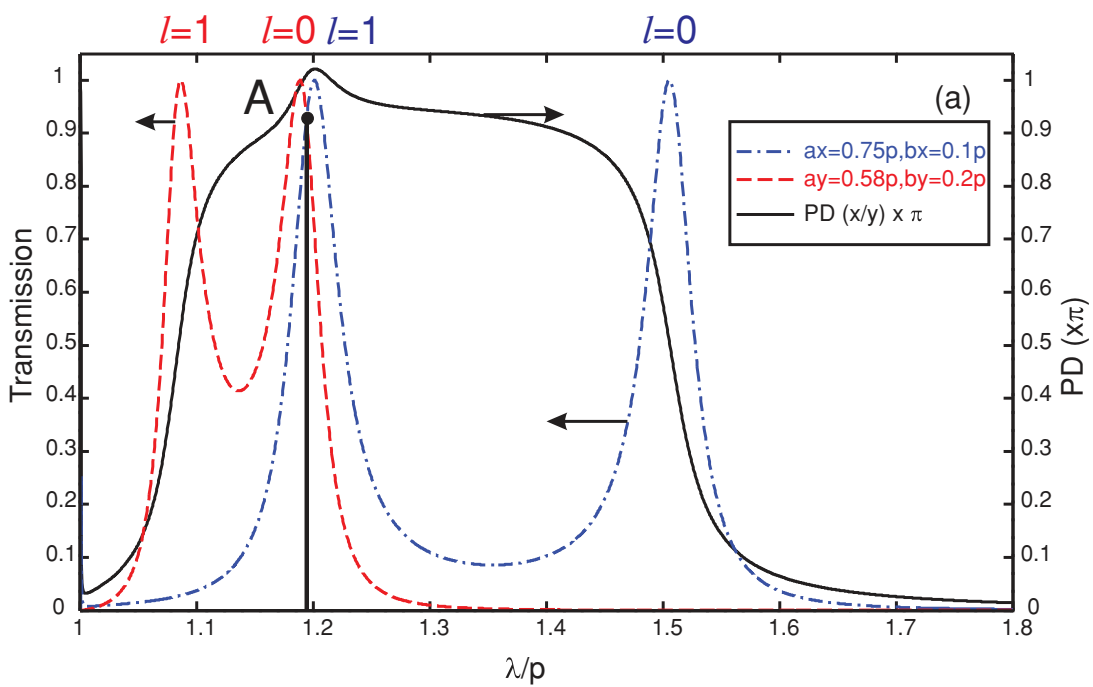

FIG. 4. (Color online) Transmission spectra for the two configurations of half-wave plate (a) and quarter-wave one (b). The geometrical parameters are given in the legend of each subfigure. The phase differences are plotted with a solid black line. The two vertical solid black lines indicates the working points for each configuration $(\lambda=1.194 p$ for the half-wave plate and $\lambda=1.182 p$ for the quarter-wave one; $h=0.8 p$ in both cases).

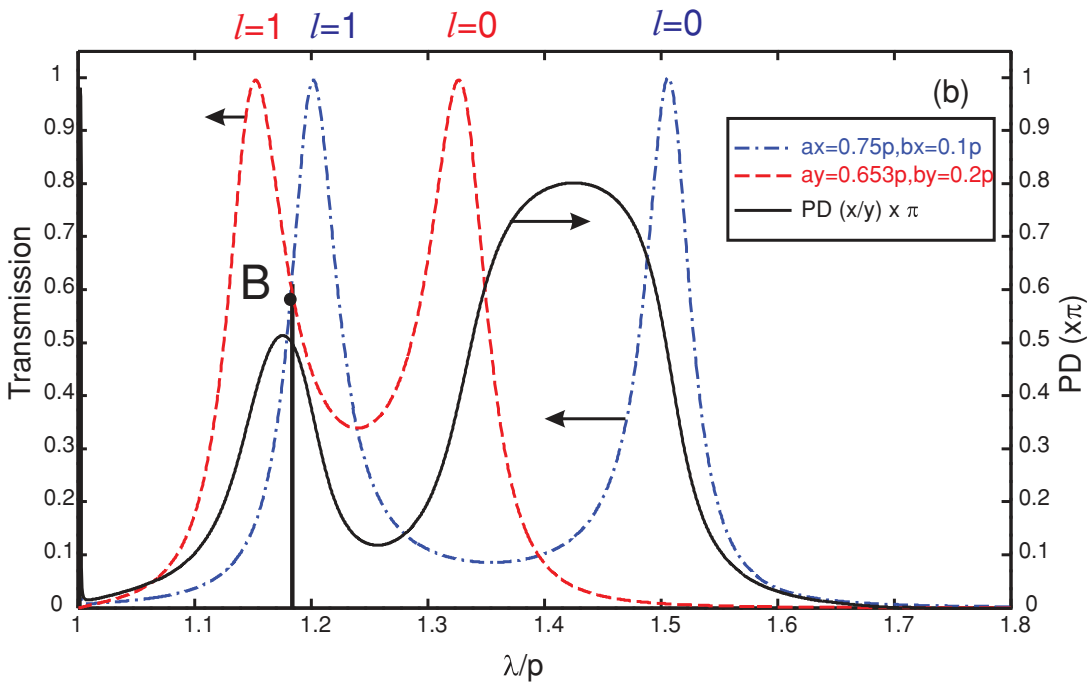




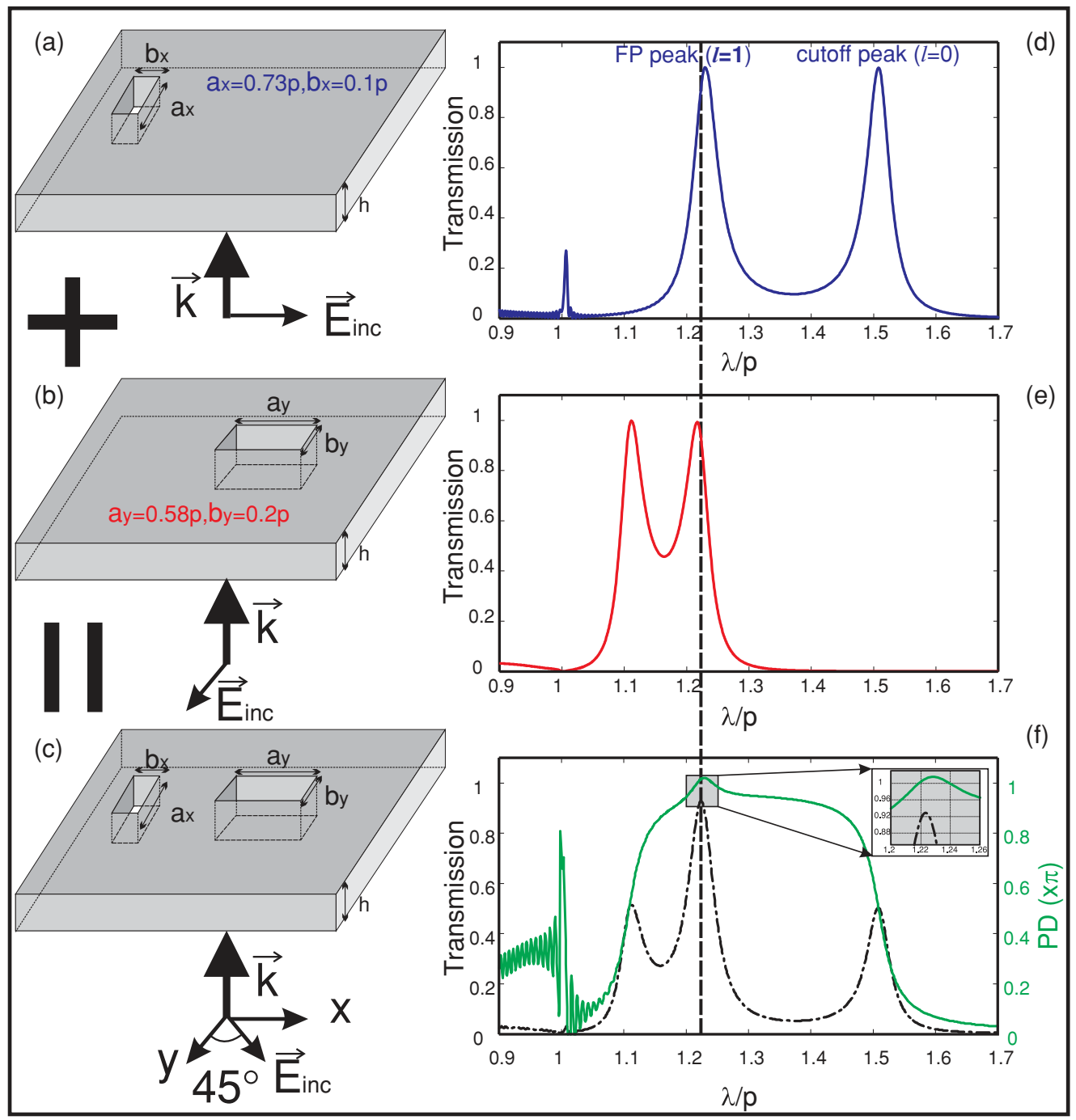

FIG. 5. (Color online) The design steps of the half-wave plate. [(a) and (d)] The schema and the transmission coefficient, respectively, of a $p$-period array of rectangular apertures when illuminated by a linearly polarized plane wave along the $x$ axis. [(b) and (e)] The corresponding schema and transmission coefficient of an array of rectangular apertures when illuminated by a $y$-linearly polarized plane wave. (c) The schema of the combined structure illuminated at $45^{\circ}$ from the $x$ axis; (f) the transmitted energy in the zero diffracted order (solid dashed black line) and the phase difference PD (solid green line). In all cases, the metal thickness is set to $h=0.83 p$.

at $45^{\circ}$ from the $x$ axis. The obtained spectrum and the PD are simultaneously plotted in Fig. 5(f). One can clearly note a $92 \%$ transmission coefficient at $\lambda=1.223 p$ accompanied by a phase difference of $\mathrm{PD}=1.01 \pi$.

In order to verify that the transmission mechanism which explains the polarization properties of the plate is well understood, we have calculated the field into the two cavities at the working wavelength value. Figure 6 presents sections of the electric intensity $\left(I=\left|E_{x}\right|^{2}+\left|E_{y}\right|^{2}+\left|E_{z}\right|^{2}\right)$ in two perpendicular planes intersecting the two apertures in their middle. The illumination conditions are the same as in Fig. 5(f). One can clearly note the presence of only one node of the intensity distribution along the $z$ direction inside the aperture $\left(a_{x}, b_{x}\right)$ while an almost uniform intensity distribution exists inside the second aperture. This confirms the excitation of the $(l=1)$ and $(l=0)$ Fabry-Perot harmonics inside the $\left(a_{x}, b_{x}\right)$ and $\left(a_{y}, b_{y}\right)$ apertures, respectively, at this wavelength.
To verify the polarization response of the designed halfwave plate, we have performed the numerical experiment shown in Fig. 7(a). So a linearly polarized incident plane wave is supposed to illuminate the plate at normal incidence. The angle of polarization $\phi$ is defined relatively to the $x$ axis. A second polarizer is used to analyze the polarization of the transmitted wave.

As is well known, after passing through a perfect halfwave plate, the original polarization plane is rotated through an angle $2 \phi$. Figure $7(\mathrm{~b})$ presents the obtained transmission spectra as a function of the analyzer angle $\alpha$ when $\phi=45^{\circ}$. At the working wavelength value $\lambda=1.223 p$, the transmission variations versus $\alpha$ almost verify a Malus law with a maximum exactly located at $\alpha=135^{\circ}=\pi-\phi$ demonstrating a rotation angle of $2 \phi$. Nevertheless, the plate is not a perfect half-wave one. The transmitted wave is elliptically polarized with a very small ellipticity of 1:3000 corresponding to a path difference of $\lambda / 2 \pm \lambda / 10000$. 


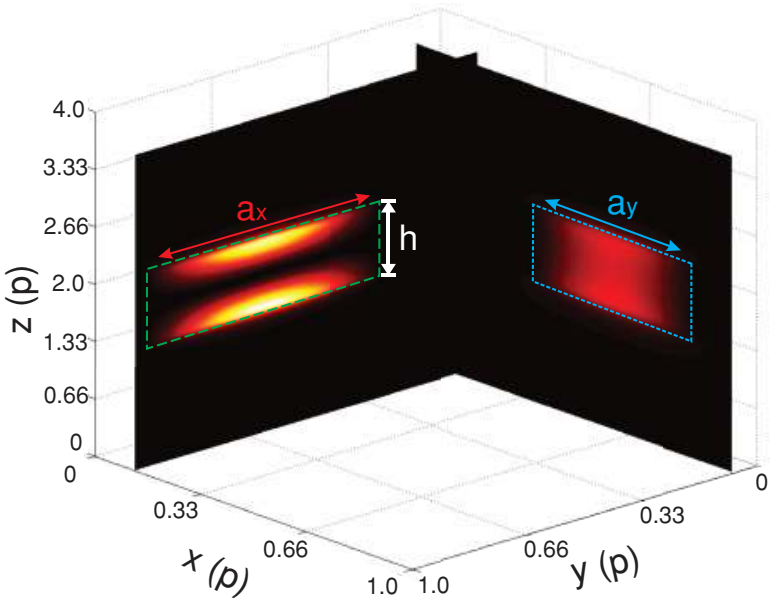

FIG. 6. (Color online) Two plane sections given the electric intensity distributions around the whole double apertured structure when illuminating at normal incidence by a linearly polarized plane wave $(\lambda=1.223 p)$. The incident electric field is directed at $45^{\circ}$ from the $x$ axis. The vertical planes intersect the apertures in their middle.

To evaluate the bandwidth of this plate, we plot on Fig. 8 the polarization ellipticity $\eta$ (solid black line) of the elliptical polarization of the wave emerging from the plate as a function of the wavelength in the case of an incident polarization at $\phi=45^{\circ}$. This ellipticity is defined by the ratio of the minor axis $A_{m}$ to the major one $A_{M}\left[\eta=\tan (\xi)=A_{m} / A_{M}\right]$. It can be related to the PD and to the amplitude of the $x$ and $y$ components through the expression:

$$
\sin (2 \xi)=\sin (2 \chi) \sin (\mathrm{PD}),
$$

where $\chi$ is given by $\tan (\chi)=E_{y} / E_{x}$.

By the same way, the ellipsis axis rotation $\beta$ (see inset of Fig. 8), that is due to the difference between the two transmitted amplitudes $E_{x}$ and $E_{y}$, is given by

$$
\tan (2 \beta)=\tan (2 \chi) \cos (\mathrm{PD}) .
$$

The variations $\beta(\lambda)$ are also plotted (dashed blue line) on the same Fig. 8 where the transmission spectrum is also remembered (dotted-dashed red line). First, we fix the wavelength bandwidth so that it corresponds to $20 \%$ variations of the transmission coefficient. According to Fig. 8, we get a bandwidth of $\Delta \lambda=\lambda / 40$ inducing a maximum elliticity variations of $\Delta \eta=0.03$. This value is very small. So it can be assumed that the polarization of the transmitted wave remains linear over all this bandwidth. In addition, the ellipsis major axis, is directed along the $y$ direction $\left(2 \times \phi=2 \times 45^{\circ}=\right.$ $90^{\circ}$ ) at the working wavelength $\lambda=1.223 p$, but this almost remains valid over all the bandwidth $\left([\Delta \beta]_{\max } \simeq 10^{\circ}\right)$. All these properties correspond to a very efficient half-wave plate.

To be more concrete, let us set the working wavelength to $\lambda_{w}=300 \mu \mathrm{m}$ that corresponds to a frequency of $1 \mathrm{THz}$. The structure dimensions become $p=245 \mu \mathrm{m}, a_{x}=179 \mu \mathrm{m}$, $a_{y}=143 \mu \mathrm{m}, b_{x}=24.5 \mu \mathrm{m}, b_{y}=49 \mu \mathrm{m}$, and $h=196 \mu \mathrm{m}$. With these values, the wavelength bandwidth is about $\Delta \lambda=$ $7.5 \mu \mathrm{m}$ that corresponds to a frequency bandwidth of $\Delta v=25 \mathrm{GHz}$.

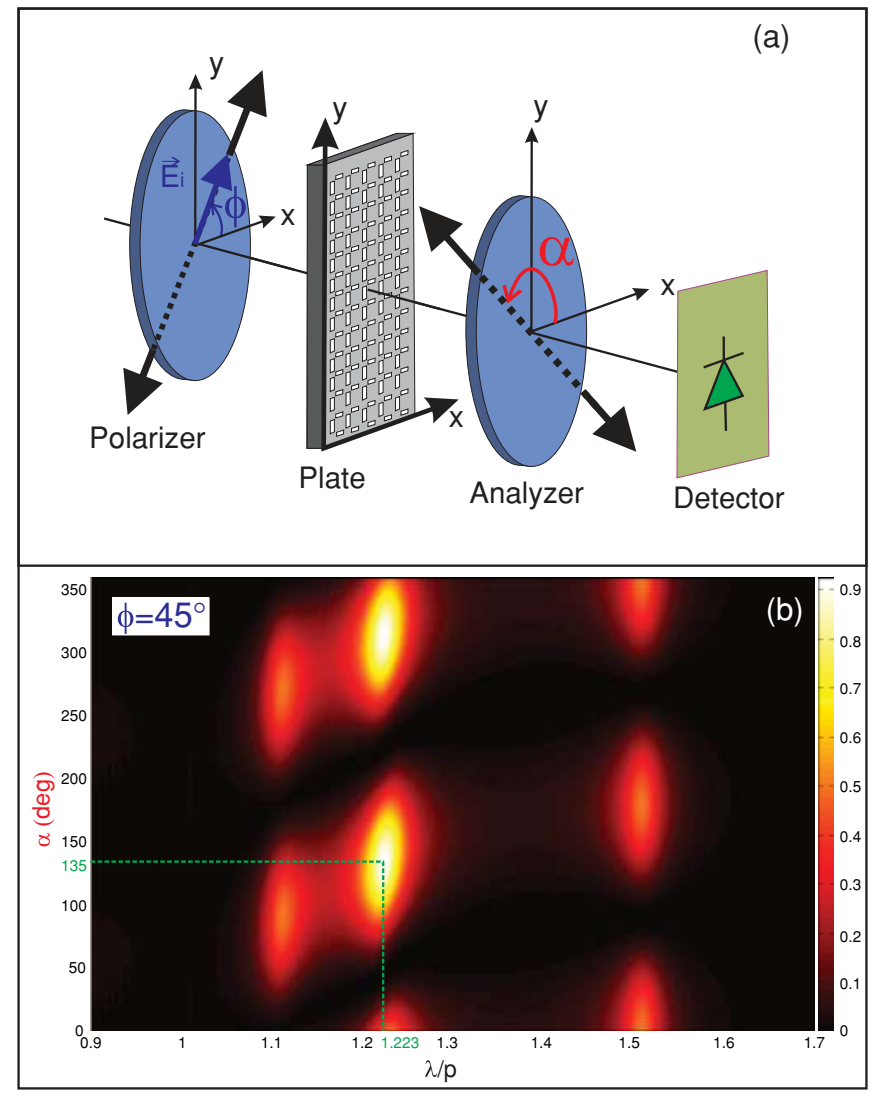

FIG. 7. (Color online) (a) Numerical experiment to verify the polarization properties of the half-wave plate. (b) Detected spectra through the analyzer versus its axis direction $(\alpha)$. The coordinates of the maximum of the detected intensity are pointed out through the dashed lines.

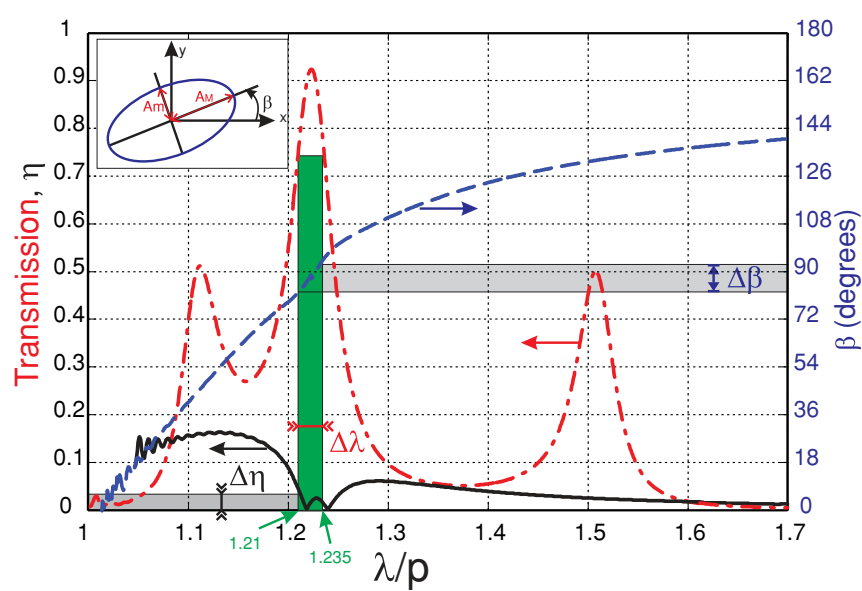

FIG. 8. (Color online) Spectrum of the ellipticity (solid black line) of the elliptical polarization emerging from the plate when illuminating at normal incidence by a plane wave that is linearly polarized at $45^{\circ}$ from the $x$ axis. The major axis position $\beta$ counted from the $x$ axis is also presented (dashed blue line). The transmission spectra (dotted-dashed red line) of Fig. 5(f) is also plotted in order to estimate the wavelength bandwidth $(\Delta \lambda)$ of the half-wave plate. 


\section{CONCLUSION}

In summary, we have designed and theoretically tested an anisotropic metamaterial based on enhanced transmission structures exhibiting guided modes inside their subwavelength apertures that are engraved into opaque metallic plates. The obtained phase difference, that can freely be set, directly involves the effective index of the guided modes inside each aperture. The geometrical parameters of the apertures (including the metal thickness) allow fixing the cut-off frequencies of the guided modes. Any phase difference value, associated with same transmission coefficients along two perpendicular polarization directions, can be obtained. An example of a halfwave plate with $92 \%$ transmission coefficient is theoretically demonstrated. The diagram of Fig. 3 shows also another solution to get a nonoptimized quarter-wave plate exhibiting a transmission coefficient of $57 \%$. This kind of structure can be exploited in both terahertz and microwave spectral domains but it also can be extended to the visible range in spite of metal losses. Nevertheless, for the proposed structure, the wavelength bandwidth where the plate can be considered as a good half-wave plate is not very large. The bandwidth depends on the width of the transmission peaks which is directly related to the width $\left(b_{x}\right.$ and $\left.b_{y}\right)$ of the apertures. It is important to optimize the plate bandwidth for each particular application. Other geometrical patterns can be used for this purpose. Their "common point" must be the high sensitivity to the polarization properties. In addition, if the technological fabrication process needs the presence of a substrate, this latter slightly modifies the transmission efficiency and induces a small modification of the working wavelength value. Consequently, an adjustment of the metal film thickness is required to reach the desired effect at the chosen wavelength. Moreover, based on the same principle, other artificial anisotropic plates can be planed and we are working on the design of quarter-wave and halfwave plates where the wavelength bandwidth is significantly improved.

\section{ACKNOWLEDGMENTS}

This work is supported by a scientific project founded by the "Région de Franche-Comté." The authors thank the SCI, the computing staff of FEMTO-ST, for technical assistance. The grant of M. Boutria is funded by educational collaboration between France and Algeria. *fbaida@univ-fcomte.fr

${ }^{1}$ J.-B. Masson and G. Gallot, Opt. Lett. 31, 256 (2006).

${ }^{2}$ S. C. Saha, Y. Ma, J. P. Grant, A. Khalid, and D. R. S. Cumming, Opt. Express 18, 12168 (2010).

${ }^{3}$ R. Gordon, A. G. Brolo, A. McKinnon, A. Rajora, B. Leathem, and K. L. Kavanagh, Phys. Rev. Lett. 92, 037401 (2004).

${ }^{4}$ J. W. Lee, M. A. Seo, D. S. Kim, J. H. Kang, and Q-Han Park, Appl. Phys. Lett. 94, 081102 (2009).

${ }^{5}$ L. D.-C. Tzuang, Y.-W. Jiang, Y.-H. Ye, Y.-T. Chang, Y.-T. Wu, and S.-C. Lee, Appl. Phys. Lett. 94, 091912 (2009).

${ }^{6}$ Benfeng Bai, Janne Laukkanen, Anni Lehmuskero, and Jari Turunen, Phys. Rev. B 81, 115424 (2010).

${ }^{7}$ T. Li, S. M. Wang, J. X. Cao, H. Liu, and S. N. Zhu, Appl. Phys. Lett. 97, 261113 (2010).

${ }^{8}$ J. Xu, T. Li, F. F. Lu, S. M. Wang, and S. N. Zhu, Opt. Express 19, 748 (2011).

${ }^{9}$ F. J. García Vidal, L. Martín Moreno, E. Moreno, L. K. S. Kumar, and R. Gordon, Phys. Rev. B 74, 153411 (2006).

${ }^{10}$ F. I. Baida and D. Van Labeke, Opt. Commun. 209, 17 (2002).

${ }^{11}$ F. I. Baida, D. Van Labeke, G. Granet, A. Moreau, and A. Belkhir, Appl. Phys. B 79, 1 (2004).

${ }^{12}$ I. A. Ibrahim, M. Mivelle, T. Grosjean, J. T. Allegre, G. W. Burr, and F. I. Baida, Opt. Lett. 35, 2448 (2010).
${ }^{13} \mathrm{~A}$ numerical verification has been made by comparing silver at $1 \mathrm{THz}\left(\varepsilon_{r}=-4.4932 \times 10^{6}+1.993 \times 10^{5} i\right)^{14}$ with a perfect conductor. The numerical difference between the two transmission spectra is less than $2 \times 10^{-3}$.

${ }^{14}$ J.-L. Coutaz, Optoélectronique térahertz (EDP Sciences, Paris, 2008).

${ }^{15}$ R. C. McPhedran and D. Maystre, Appl. Phys. 14, 1 (1977).

${ }^{16}$ A. Roberts and R. C. McPhedran, IEEE Trans. Antennas Propag. 36, 607 (1988).

${ }^{17}$ F. J. García Vidal, L. Martín Moreno, T. W. Ebbesen, and L. Kuipers, Rev. Mod. Phys. 82, 729 (2010).

${ }^{18} \mathrm{Ph}$. Lalanne, J. P. Hugonin, S. Astilean, M. Palamaru, and K. D. Möller, J. Opt. A: Pure Appl. Opt. 2, 48 (2000).

${ }^{19}$ J. T. Shen and P. M. Platzman, Phys. Rev. B 70, 035101 (2004).

${ }^{20}$ J. T. Shen, P. B. Catrysse, and S. Fan, Phys. Rev. Lett. 94, 197401 (2005).

${ }^{21}$ J. Jung, L. Martín Moreno, and F. J. García Vidal, New J. Phys. 11, 123013 (2009).

${ }^{22}$ D. H. Dawes, R. C. McPhedran, and L. B. Whitbourn, Appl. Opt. 28, 3498 (1989).

${ }^{23}$ W. Fan, S. Zhang, B. Minhas, K. J. Malloy, and S. R. J. Brueck, Phys. Rev. Lett. 94, 033902 (2005).

${ }^{24}$ Y. Poujet, J. Salvi, and F. I. Baida, Opt. Lett. 32, 2942 (2007).

${ }^{25}$ A. Belkhir, O. Arar, S. S. Benabbes, O. Lamrous, and F. I. Baida, Phys. Rev. E 81, 046705 (2010). 